\title{
Nonlinear response of tropical lower-stratospheric temperature and water vapor to ENSO
}

\section{Chaim I. Garfinkel et al.}

Correspondence to: Chaim I. Garfinkel (chaim.garfinkel@mail.huji.ac.il)

The copyright of individual parts of the supplement might differ from the CC BY 3.0 License. 


\section{Linearity of the response of the BDC to ENSO}

The text argues that the nonlinearity in the lower stratospheric response to ENSO arises not from the large scale stratospheric $\mathrm{BDC}$, but rather from processes below in the tropical tropopause layer. We now provide additional support for the linearity of the large scale stratospheric BDC to ENSO. Figure S2 and S3 shows the response in tropical mean age at 50hPa and 70hPa and $\mathrm{NH}$ polar cap temperature at $85 \mathrm{hPa}$ to ENSO in the coupled ocean-atmosphere and AGCM experiments respectively. The response of mean age is linear with respect to ENSO in all seasons at these levels. The NH polar stratospheric response to ENSO is also linear in these experiments, and displays little sensitivity to the flavor of the EN event: both CP and EP EN events lead to polar stratospheric warming (in agreement with Garfinkel et al., 2013). There is little difference in the response between the coupled ocean-atmosphere simulations and the AGCM simulations.

10 These changes in the BDC are ultimately driven by upward propagating waves from the troposphere driven by ENSO, and the changes in these waves are diagnosed by examining the 100hPa heat flux response to ENSO (see Figure S4 for the AGCM simulations; the daily data to compute the heat flux for the coupled integrations is not available). We average the heat flux from $25^{\circ}$ to $75^{\circ}$ in each hemisphere following Fueglistaler et al. (2014), and the SH is shown in the left column and the NH is shown in the middle column. In both hemispheres heat flux increases during EN (in the SH heat flux is negative climatologically), and the changes in heat flux are also linear with respect to ENSO. The right column of Figure S4 shows the wavenumber 1 heat flux from $40^{\circ}$ to $80^{\circ}$, the wave driving of most importance for the polar warming in response to EN (Garfinkel and Hartmann, 2008). It too changes linearly in response to ENSO.

The net effect is that EN leads to more wave driving of the BDC, leading to an accelerated BDC and cooling of the tropical lower stratosphere via enhanced adiabatic cooling. This adiabatic cooling must be balanced by anomalous diabatic heating, as

20 the thermodynamic budget must balance. The way in which this balance occurs is through anomalous longwave heating. To confirm this, Figure S5 compares the diabatic heating due to longwave and shortwave radiation. The temperature and diabatic heating for the coupled ocean-atmosphere integration is shown in Figure 2 of the main text. The colder temperatures present during EN emit less radiation, and thus the usual longwave cooling is now weaker than before. This manifests as a positive longwave heating anomaly. Quantitatively, the slope in total diabatic heating and from longwave diabatic heating is nearly identical, though the slope for longwave is slightly larger. It does not appear to matter which flavor of ENSO event is occurring (red, black, and blue markers all line up). The slope is largest in JF, likely because the subpolar NH heat flux is affected most strongly in JF (right column of Figure S4). EN causes the shallow branch to accelerate in all seasons (Simpson et al., 2011).

The second column of figure S5 shows shortwave diabatic heating. For shortwave, EN leads to a slight cooling of the atmosphere. This could be because EN leads to less ozone (by $\sim 5 \%$ per K due to an accelerated BDC as found by Oman et al.

30 (2013)), and hence there is less shortwave heating. However, this effect is far smaller than the longwave effect, so it is of secondary importance. However, it could explain the slight difference in slope between DTDT and DTDTLW, as the slopes for DTDTLW and DTDTSW (left and middle of figure S5) add up to that for DTDT (middle of Figure 2 of the main text). 


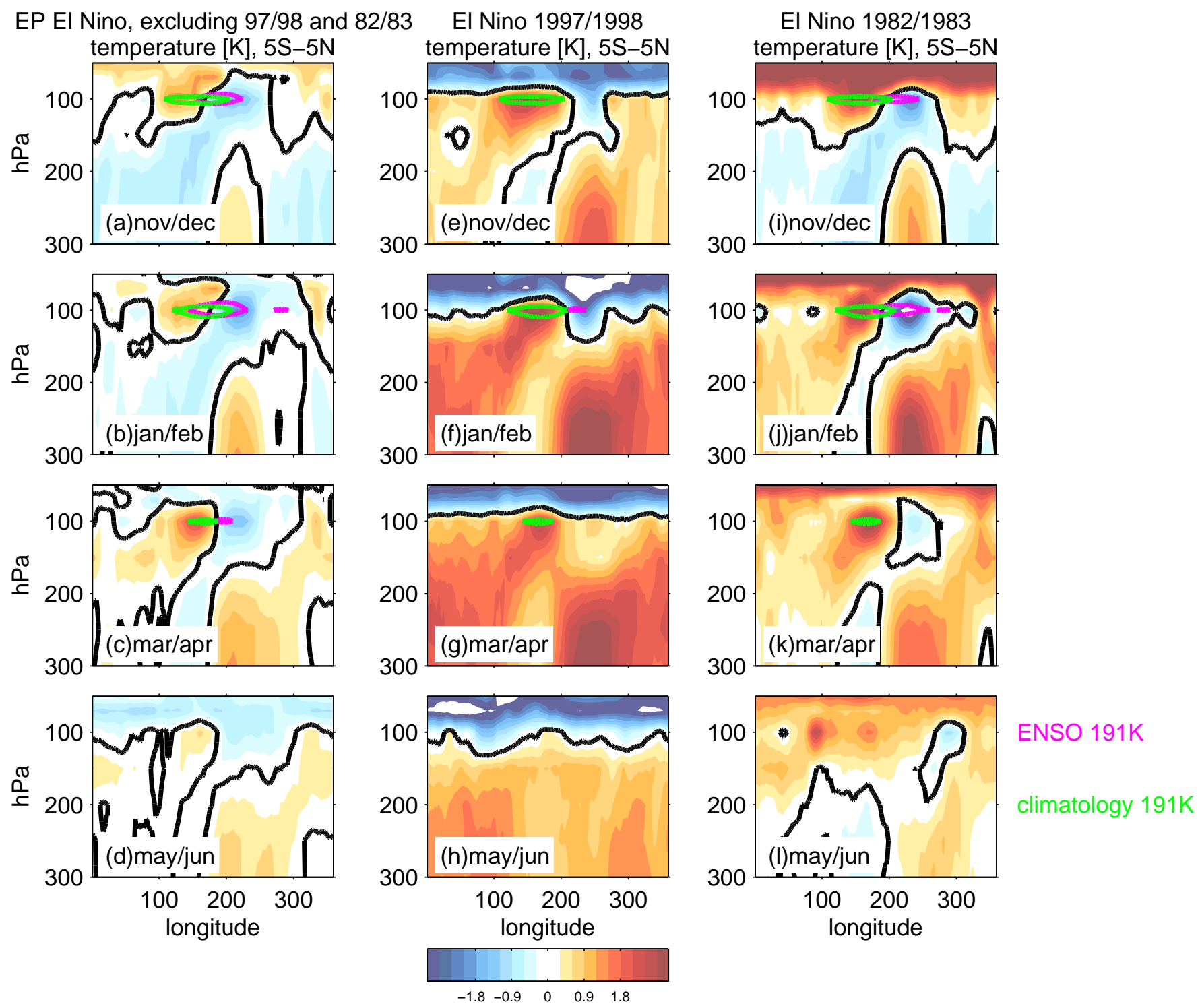

Fig. S 1. As in figure 8 in the main body but for the MERRA. Note that the 1982/1983 EN was preceded by the eruption of El Chicon, and thus the stratosphere is anomalously warm in the right column. 

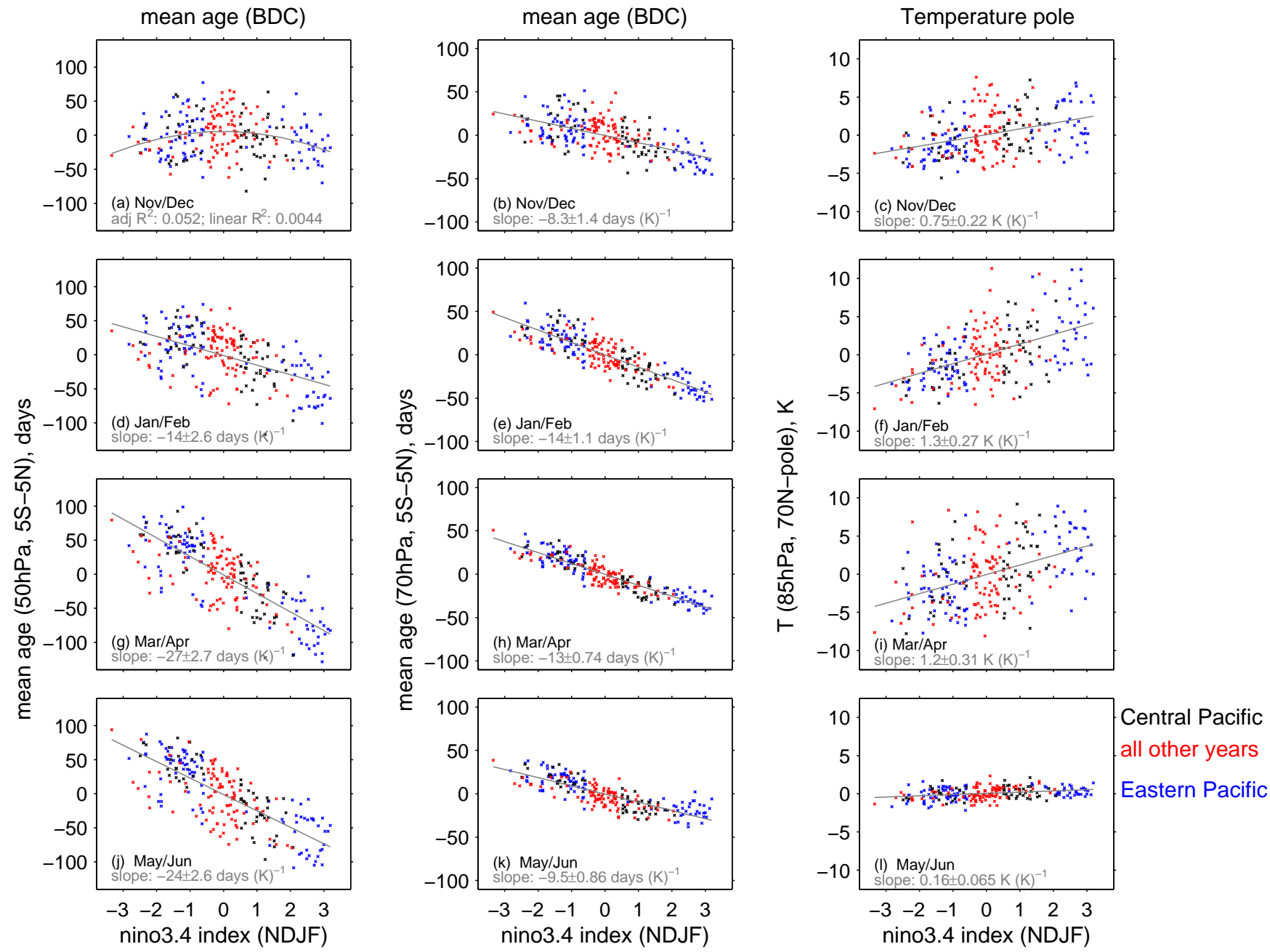

Fig. S 2. As in Figure 2 of the main text but for anomalies in mean age at 50hPa and $70 \mathrm{hPa}$ (left and center) and NH polar cap temperature changes at $85 \mathrm{hPa}$ (right). 

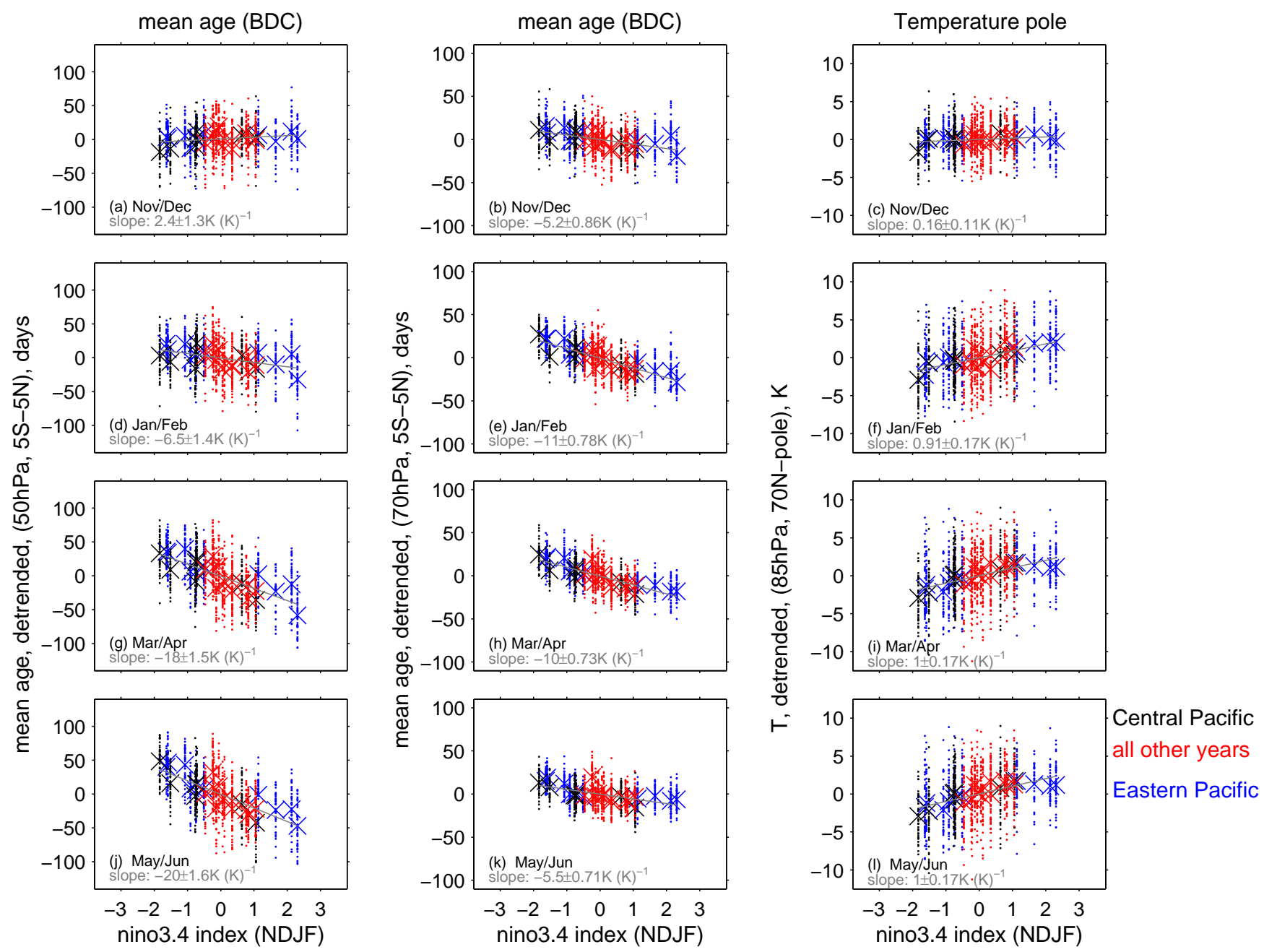

Fig. S 3. As in Figure S2 but for 42 AGCM GEOSCCM integrations. The ensemble mean for each EN event is indicated with a large $\mathrm{x}$. 

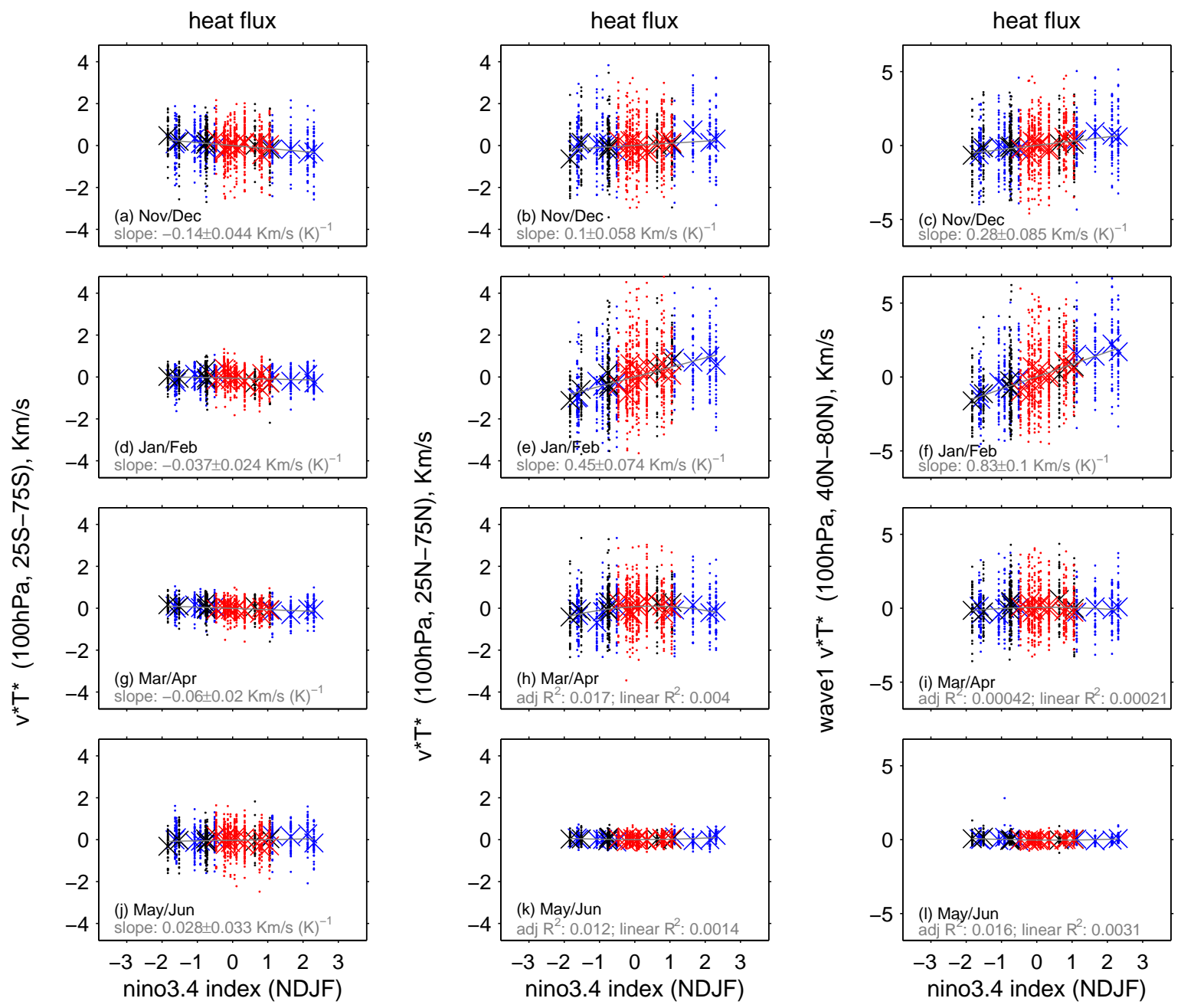

Fig. S 4. Heat flux anomalies for ENSO events in the AGCM GEOSCCM run. The left column is for SH heat flux area weighted from 25S to $75 \mathrm{~S}$ (note that negative values indicate more heat flux in the $\mathrm{SH}$ ), the middle column is for $\mathrm{NH}$ heat flux area weighted from $25 \mathrm{~N}$ to $75 \mathrm{~N}$, and the right column is for $\mathrm{NH}$ zonal wavenumber 1 heat flux area weighted from $40 \mathrm{~N}$ to $80 \mathrm{~N}$. 

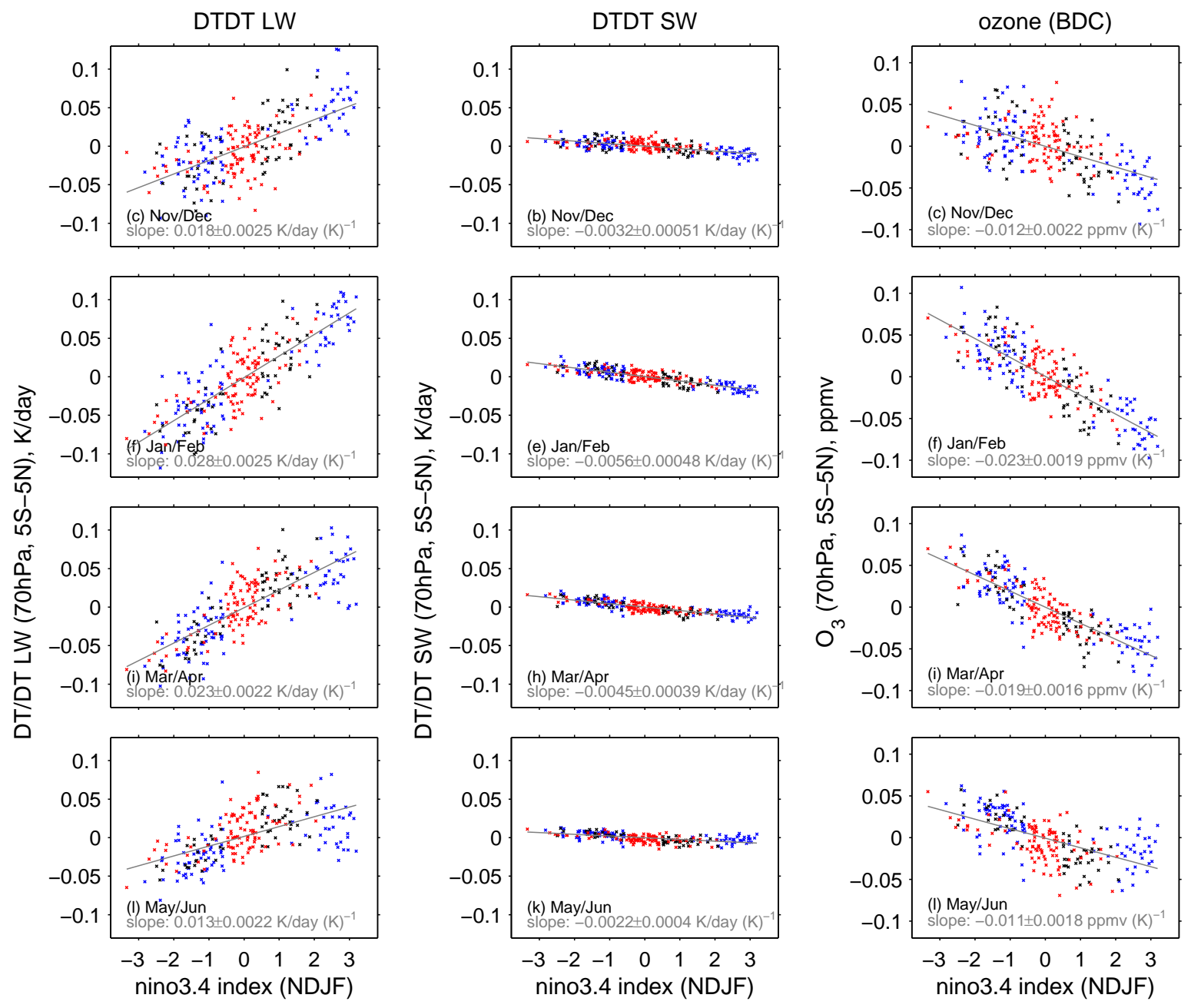

Fig. S 5. BDC anomalies for ENSO events in the last 240 years of a coupled ocean-atmosphere run. 
Acknowledgements. CIG was supported by the Israel Science Foundation (grant number 1558/14) and by a European Research Council starting grant under the European Union's Horizon 2020 research and innovation programme (grant agreement No 677756). We thank those involved in model development at GSFC-GMAO, and support by the NASA MAP program. We thank Valentina Aquila for performing some of the experiments discussed here, and Darryn W Waugh and Margaret M Hurwitz for suggestions. High-performance computing 5 resources were provided by the NASA Center for Climate Simulation (NCCS). Correspondence and requests for data should be addressed to C.I.G. (email: chaim.garfinkel@mail.huji.ac.il). El Niño indices based on the ERSSTv4 data were downloaded from cpc.ncep.noaa.gov/data/indices/ersst4.nino.mth.81-10.ascii. 


\section{References}

Fueglistaler, S., Abalos, M., Flannaghan, T., Lin, P., and Randel, W.: Variability and trends in dynamical forcing of tropical lower stratospheric temperatures, Atmospheric Chemistry and Physics, 14, 13 439-13 453, doi:10.5194/acp-14-13439-2014, 2014.

Garfinkel, C. I. and Hartmann, D. L.: Different ENSO Teleconnections and Their Effects on the Stratospheric Polar Vortex, J. Geophys. Res.Atmos., 113, doi:10.1029/2008JD009920, 2008.

Garfinkel, C. I., Hurwitz, M. M., Waugh, D. W., and Butler, A. H.: Are the Teleconnections of Central Pacific and Eastern Pacific El Niño Distinct in Boreal Wintertime?, Climate Dynamics, pp. 1-18, doi:10.1007/s00382-012-1570-2, 2013.

Oman, L. D., Douglass, A. R., Ziemke, J. R., Rodriguez, J., Waugh, D. W., and Nielsen, J. E.: The ozone response to ENSO in aura satellite measurements and a chemistry-climate simulation, J. Geophys. Res., 118, 965-976, doi:10.1029/2012JD018546, 2013.

10 Simpson, I. R., Shepherd, T. G., and Sigmond, M.: Dynamics of the lower stratospheric circulation response to ENSO, Journal of the Atmospheric Sciences, 68, 2537-2556, 2011. 